\title{
The thorax tomography correlation in COVID-19 RT-PCR positive patients
}

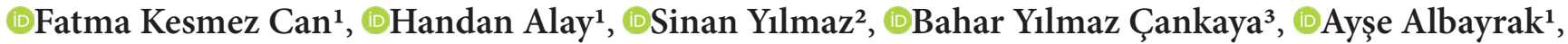

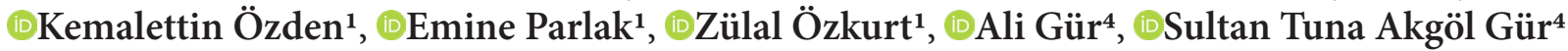 \\ ${ }^{1}$ Atatürk University, School of Medicine, Department of Infectious Diseases and Clinical Microbiology, Erzurum, Turkey \\ ${ }^{2}$ Atatürk University, School of Medicine, Department of Public Health, Erzurum, Turkey \\ ${ }^{3}$ Atatürk University, School of Medicine, Department of Radiology, Erzurum, Turkey \\ ${ }^{4}$ Atatürk University, School of Medicine, Department of Emergency Medicine, Erzurum, Turkey
}

Cite this article as: Kesmez Can F, Alay H, Yulmaz S, et al. The thorax tomography correlation in COVID-19 RT-PCR positive patients. Anatolian Curr Med J 2021; 3(2); 145-150.

\begin{abstract}
Aim: Although the RT-PCR test of pharyngeal swabs is the gold standard for diagnosing coronavirus disease 2019 (COVID-19), radiological imaging techniques, particularly thoracic computerized tomography (CT) were also used frequently as needed during the pandemic. The aim of this study is to investigate thorax CT findings in patients with COVID-19 confirmed by RT-PCR and to evaluate its relationship with clinical features.

Material and Method: This study included 311 consecutive patients who were hospitalized between April 1, 2020, and June 1, 2020, with COVID-19 diagnosis based on RT-PCR (+) results and underwent a thorax CT within 24-48 hours of admission. Symptoms, clinical status, co-morbidities of the patients were evaluated. Thorax CT findings were assessed by the Department of Radiology and the results were analyzed in relation to the clinical status of the PCR $(+)$ patients.

Results: The study group consisted of 170 male (57.7\%) and 141 female (42.3\%) patients with mean age $46.7 \pm 33.7$ years. Among the COVID-19 cases, 51 (16.4\%) were asymptomatic, the clinical course was mild-moderate in 197 (63.3\%) and severe in 63(20.3\%). During follow-up 21 (6.8\%) required intensive care and $10(3.2 \%)$ died. The most common symptoms observed were cough $(33.4 \%)$, weakness $(30.2 \%)$ and fever (28\%). The most commonly encountered co-morbidity in COVID-19 patients was hypertension (10.3\%) followed by diabetes mellitus (7.7\%), coronary artery disease (5.1\%). Thorax CT findings were assessed as normal in $21.9 \%$ of the patients; viral pneumonia was detected in $20.9 \%$ and $27.7 \%$ were reported as compatible with COVID-19. Bilateral involvement was seen on CT scan in $49.2 \%$ of the patients. In regard to thorax CT imaging characteristics that suggest COVID-19 disease, the most common was ground-glass opacities observed in 181 (27.2\%) and the least common was a vascular enlargement in $4(0.6 \%)$ of the patients.

Conclusion: COVID-19 is an air-borne disease that primarily affects the lungs. Thus, it is essential to define radiological lung involvement. The common CT findings of COVID-19 disease are similar to other viral pulmonary infections. The clinicians being familiar with common imaging features of COVID-19 would contribute to earlier detection and thus reduced mortality associated with the disease.
\end{abstract}

Keywords: Computed tomography, COVID-19, RT-PCR, emergency, infection

\section{INTRODUCTION}

The Coronavirus disease, which is a severe acute respiratory syndrome caused by a highly contagious coronavirus 2 (SARS-CoV-2), was reported firstly in Wuhan, State of Hubei, China in December 2019. The disease caused by this virus was defined as "Coronavirus Disease 2019 (COVID-19)" by the World Health Organization (WHO). The disease soon took hold of the whole world and the WHO announced that the disease progressed to become a pandemic on March 11, 2020 (1). Up to date, six coronavirus types known to cause infectious disease in humans have been defined. Among these, 229E, OC43, NL63, and HKU1 infections primarily cause common cold symptoms and have a mild course.
The other two types including Severe Acute Respiratory Syndrome Coronavirus (SARS-CoV) and the Middle East Respiratory Syndrome Coronavirus (MERS-CoV) cause severe disease (2). SARS-CoV-2 is the seventh coronavirus known to infect humans. The clinical spectrum of the SARS-CoV-2 infection includes a wide range of presentations from asymptomatic infection, mild upper respiratory infection to severe viral pneumonia leading to respiratory failure, multi-organ failure, and even death $(3,4)$. The most common clinical symptoms are fever, cough, sore throat, headache, weakness, muscle pain, and shortness of breath. These symptoms are not specific to the disease and thus rapid diagnosis requires 
tests and imaging assessment (5). The gold standard for COVID-19 diagnosis is the detection of SARS-CoV-2 RNA by reverse transcriptase-polymerase chain reaction (RT-PCR) from nasopharyngeal or oropharyngeal swab specimens. On the other hand, if the RT-PCR is negative and clinic features are highly suspicious for the disease, repeating RT-PCR test is recommended $(6,7)$. It was reported that thorax computerized tomography is a relevant method for diagnosis, pneumonia grading, and disease monitorization. However, thorax CT is not a screening test for COVID-19 (8). Considering overlapping CT findings in respiratory infections due to various etiologies, thorax CT findings have high sensitivity (97\%) but low selectivity (25\%) (9). The most common CT findings in COVID-19 cases include ground-glass opacities, consolidations, reticular pattern, cobblestone pattern, vascular enlargement, nodules, cavitation, lymph node enlargement, and pleural effusion $(10,11)$. The aim of this study was to describe CT findings in relation to the clinical course in patients with RT-PCR confirmed COVID-19 infection.

\section{MATERIAL AND METHOD}

This study was designed and submitted to the T.R. Ministry of Health COVID-19 Scientific Research Committee and Ataturk University Medical School Clinical Research Ethics Committee for approval. Approval was granted by both committees (ethical approval no: B.30.2.ATA.0.01.00/276). All procedures were performed adhered to the ethical rules and the Helsinki Declaration of Principles.

\section{Patient Groups}

In this descriptive study, 311 consecutive patients who admitted to Ataturk University Medical School Research Hospital between April 1, 2020, and June 1, 2020, with a positive COVID-19 RT-PCR test, hospitalized at the COVID-19 clinic and underwent a thorax CT scan were included. The patients were divided into three groups based on their clinical status.

Group 1 asymptomatic: Asymptomatic patients found to be RT-PCR positive after high-risk contact.

Group 2 mild-moderate pneumonia: Includes patients with symptoms including fever, myalgia/arthralgia, cough, and sore throat, respiration rate $<30 /$ minute, $\mathrm{SpO}_{2}$ level $>90 \%$ on room air, and mild-moderate pneumonia $(<50 \%)$ findings on tomography.

Group 3 severe pneumonia: Includes patients with symptoms including fever, myalgia/arthralgia, cough, and sore throat, tachypnea (respiration rate $\geq 30$ /minute), $\mathrm{SpO}_{2}$ level $\leq 90 \%$ on room air and, bilateral extensive pneumonia $(>50 \%)$ findings on tomography.

Unenhanced thorax CT scans were performed on all patients in the supine position using a 256 slice CT device (Aquillon; Toshiba Medical Systems, Tokyo, Japan). The tomography findings were assessed by the Department of Radiology. Patients over 18 years of age were included in the study. The clinical status and co-morbidities of the patients were evaluated. The distribution and patterns of lung lesions were assessed by subgroups. The thorax tomography findings of patients with RT-PCR (+) SARS-CoV-2 infection were evaluated.

\section{Statistical Analysis}

The data were analyzed using the Statistical Package for the Social Sciences (SPSS v20) software. Categorical variables were presented as number and percent, numerical variables were presented as mean and standard deviation.

\section{RESULTS}

The study included RT-PCR confirmed 311 COVID-19 patients aged 18-77 years. The study group consisted of 170 male $(57.7 \%)$ and 141 female (42.3\%) patients with mean age $46.7 \pm 33.7$ years. Among the COVID-19 cases, 51 (16.4\%) were asymptomatic, the clinical course was mildmoderate in 197 (63.3\%) and severe in 63 (20.3\%). During follow-up 21 (6.8\%) required intensive care and $10(3.2 \%)$ died. The most common symptoms observed were cough $104(33.4 \%)$, weakness 94 (30.2\%) and fever 87 (28\%). The most commonly encountered co-morbidity in COVID-19 patients was hypertension (10.3\%) (Table 1).

\section{Table 1. Distribution of symptoms, co-morbidities and clinical} features of COVID-19 patients

\begin{tabular}{|lcc|}
\hline Symptoms & $\mathbf{n}$ & \% \\
\hline Cough & 104 & 33.4 \\
Weakness & 94 & 30.2 \\
Fever & 87 & 28.0 \\
Dyspnea & 55 & 17.7 \\
Sore throat & 35 & 11.3 \\
\hline Abdominal pain & 26 & 8.4 \\
Loss of appetite & 14 & 4.5 \\
Loss of taste/smell & 13 & 4.2 \\
Diarrhea & 9 & 2.9 \\
Nausea/vomiting & 5 & 1.6 \\
Co-morbidities & & \\
HT & 32 & 10.3 \\
DM & 24 & 7.7 \\
CAD & 16 & 5.1 \\
COPD & 15 & 4.8 \\
Clinic & & \\
Asymptomatic & 51 & 16.4 \\
\hline Mild/moderate & 197 & 63.3 \\
Severe & 63 & 20.3 \\
Intensive care & & \\
Yes & 21 & 6.8 \\
No & 290 & 93.2 \\
Mortality & & \\
Yes & 10 & \\
No & 301 & \\
\hline HT; Hypertension, DM; Diabetes mellitus, CAD; Coronary artery disease, COPD; \\
Chronic obstructive pulmonary disease & & \\
\hline
\end{tabular}


Thorax CT findings were assessed as normal in 21.9\% of the patients while viral pneumonia was detected in $20.9 \%$ and $27.7 \%$ was reported as compatible with COVID-19. Multiple findings were observed in some of the CT scans. In total, the detected CT findings were ground-glass opacities in $181(27.2 \%)$, bilateral involvement in 153 (23.0\%), peripheral localization in $80(12.0 \%)$ (Figure 1), consolidation in $74(11.1 \%)$, LAP/cavity/nodule in 50 (\%7.5) (Figure 2-3), band formation in $56(8.4 \%)$, cobblestone appearance in $22(3.3 \%)$ (Figure 4), reticular pattern in $17(2.6 \%)$, pleural thickening in $14(2.1 \%)$, pleural effusion in 9 $(1.4 \%)$, bronchiectasis changes in $5(0.8 \%)$ and vascular enlargement in 4 (0.6\%) (Figure 5) (Table 2).
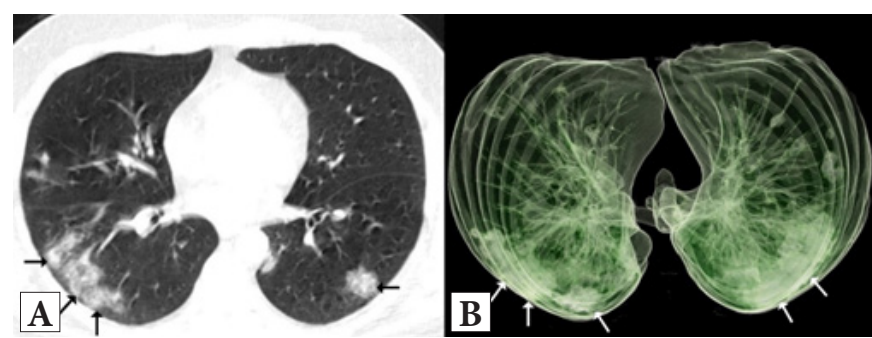

Figure 1. 46-year-old male patient who admitted with cough and back pain. The thorax CT axial parenchymal window (A) and threedimensional pulmonary image (B) show multiple ground-glass density nodules (arrows) on lower lobes bilaterally.

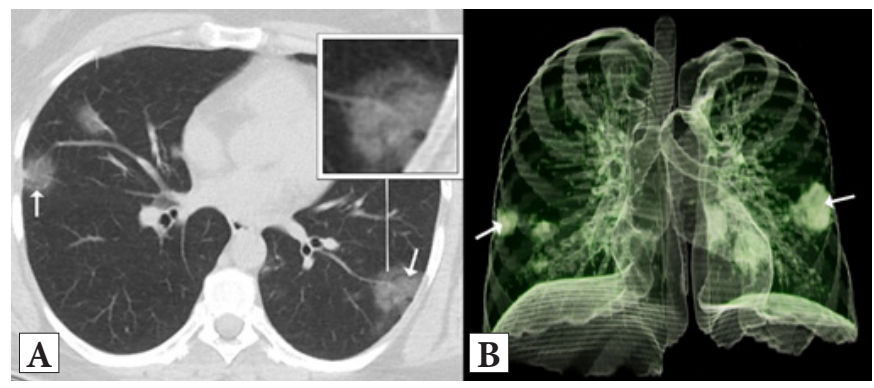

Figure 2. 46-year-old female patient who admitted with fever, generalized body pain and back pain. No co-morbidities reported. The thorax CT axial parenchymal window image (A) shows bilateral ground-glass density nodules (arrows). Three-dimensional chest CT image of the nodules (B)

\begin{tabular}{|c|c|c|}
\hline Thorax CT Findings & $\mathbf{n}$ & $\%$ \\
\hline Grounded glass & 181 & 27.2 \\
\hline Bilateral involvement & 153 & 23.0 \\
\hline Peripheral involvement & 80 & 12.0 \\
\hline Consolidation & 74 & 11.1 \\
\hline Band formation & 56 & 8.4 \\
\hline LAP/cavity/nodular pattern & 50 & 7.5 \\
\hline Cobblestone appearance & 22 & 3.3 \\
\hline Reticular pattern & 17 & 2.6 \\
\hline Pleural thickening & 14 & 2.1 \\
\hline Pleural effusion & 9 & 1.4 \\
\hline Bronchiectasis changes & 5 & 0.8 \\
\hline Vascular enlargement & 4 & 0.6 \\
\hline
\end{tabular}

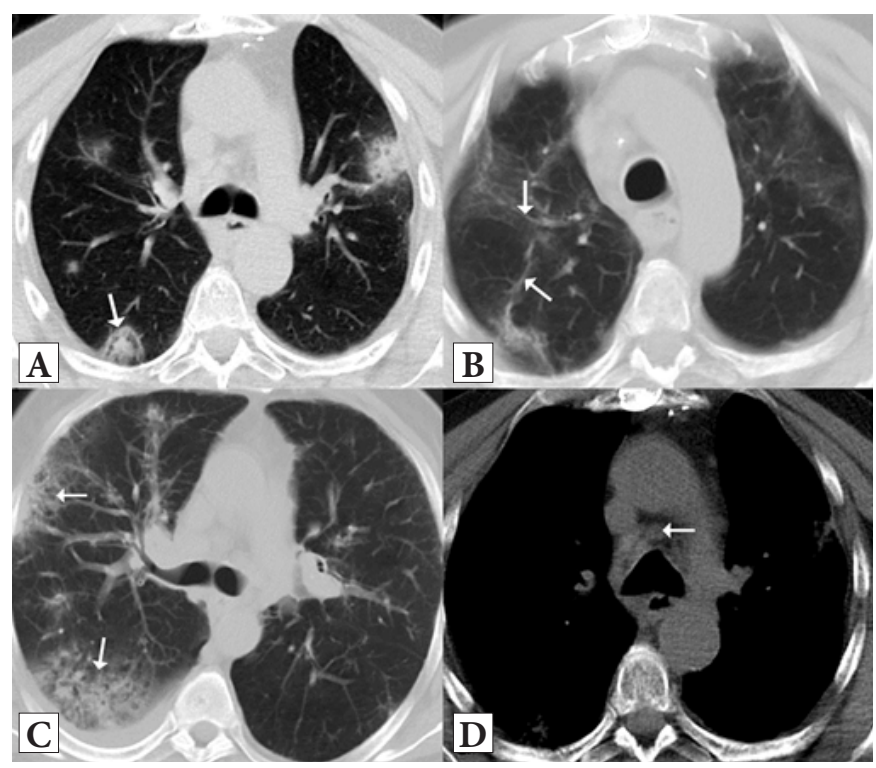

Figure 4. 72-year-old male patient who admitted with fever and cough had history of hypertension and coronary artery disease. The patient required intensive care during follow-up and was discharged following treatment in intensive care unit. On admission, thorax CT axial parenchymal window image (A) revealed nodular density with reverse halo adjacent to the pleura in right pulmonary lower lobe (arrow). At follow-up on Day 10, axial parenchymal window CT (B) showed diffuse ground-glass appearance in upper lobes bilaterally and fibrotic band formation on the right (arrow) and in lower

sections $(\mathbf{C})$ cobblestone appearance in right pulmonary middle and lower lobes (arrows) and lymph node in aortic-pulmonary area with mediastinal dose (D).

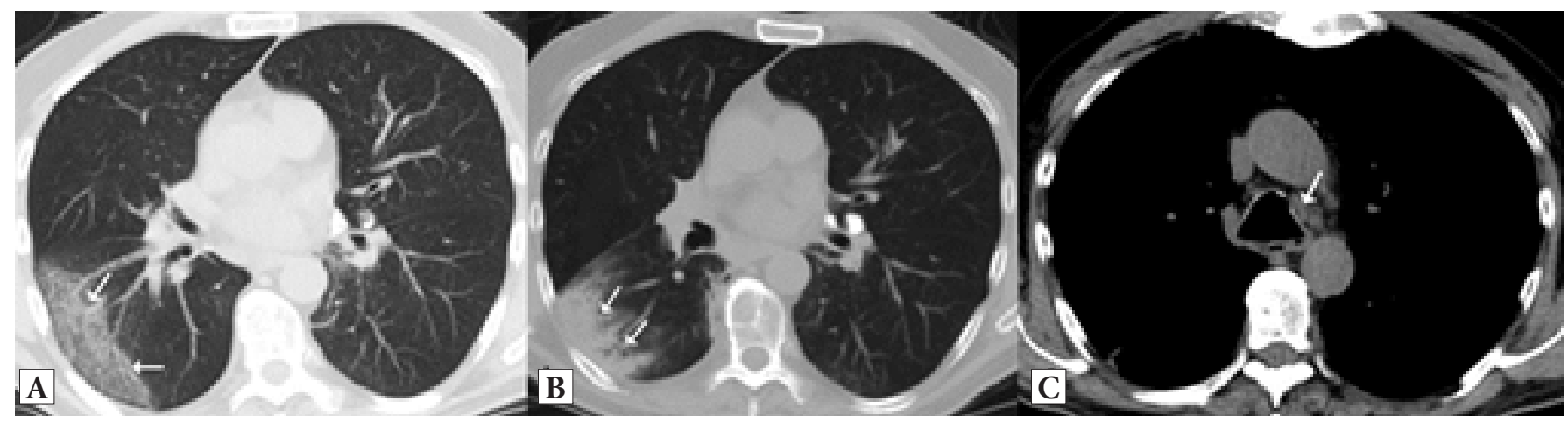

Figure 3. 50-year-old male patient with no known chronic disease admitted with a 5-day history of fever, sore throat and back pain. Thorax CT axial parenchymal window image (A) shows reticular-nodular densities adjacent to the pleura in right lower lobe and accompanying ground-glass appearance (arrows). Follow-up axial parenchymal window image at 1 week (B) shows consolidation area with air bronchogram at the same localization (arrows). Lymph node is observed in aortic-pulmonary window (arrow) on axial CT section with mediastinal dose $(\mathbf{C})$ 


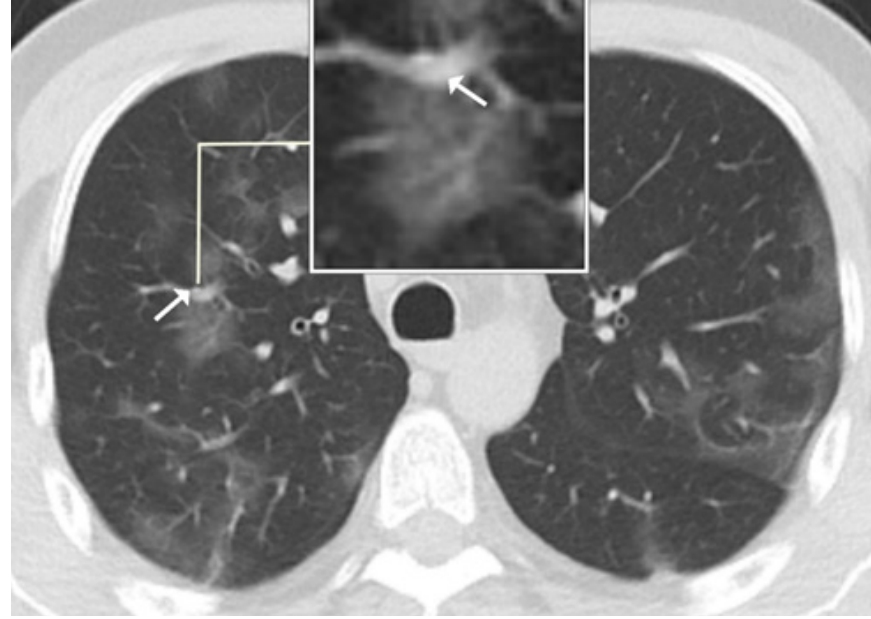

Figure 5. 47-year-old male patient who admitted with fever and back pain. The thorax CT axial parenchymal window image showed bilateral ground-glass appearance bilaterally and on the right, vascular enlargement adjacent to the ground-glass density (arrow).

\section{DISCUSSION}

Early recognition of COVID-19 is essential for treatment and disease control. The definitive diagnosis is possible by RT-PCR testing of respiratory specimens. In early cases, while tests are still negative and the patient is asymptomatic, thorax CT can reveal some findings which allow early detection of the disease.

In this study, among the 311 RT-PCR positive patients 51 (16.4\%) were asymptomatic which suggests the absence of clinical symptoms cannot rule out the COVID-19 infection. In another study conducted with 90 patients, $7 \%$ were found to be asymptomatic (12). In asymptomatic patients, pulmonary findings appear to be less frequent and mild. On the contrary, symptomatic patients seem to admit with prominent consolidations, more generalized parenchymal involvement and pulmonary signs (13). In the study population, 197 (63.3\%) had mild/moderate pneumonia and 63 (20.3\%) had severe pneumonia. In a study conducted in China, the clinical presentation was mild in $80 \%$ and severe in $15 \%$ of the cases while $5 \%$ had highly severe conditions with high mortality such as respiratory failure, shock, organ failure; the reported mortality rate was $2.3 \%$ (14). Among our patients, $21(6.8 \%)$ required intensive care; $10(3.2 \%)$ died, all from the seriously ill patient group. The clinical status and prognosis data of our study were compatible with the literature. Cardiovascular diseases, diabetes, hypertension, chronic pulmonary diseases, chronic renal failure, obesity, cancer, immune-suppression conditions, chronic liver disease and smoking were reported as factors that can affect the severity of COVID-19 disease $(15,16)$. In this study, the most commonly encountered co-morbidity was hypertension in $32(10.3 \%)$ followed by diabetes in $24(7.7 \%)$, coronary artery disease in 16 (5.1\%). The commonly reported symptoms were cough in $104(33.4 \%)$, weakness in $94(30.2 \%)$, fever in $87(28 \%)$, dyspnea in 55 (17.7\%) patients (Table 1). In previous studies, fever and cough were reported most frequently followed by weakness, myalgia and dyspnea $(17,18)$.

The involvement in COVID-19 pneumonia is similar to other viral pneumonia. In this study, among the CT scans $20.9 \%$ were reported as viral pneumonia and $27.7 \%$ as compatible with COVID-19 disease with atypical findings. The thorax CT was evaluated as normal in $21.9 \%$ of the patients. Particularly in early stage of the disease, it appeared thorax CT could be normal and this would not exclude the disease. The evaluation based on overall tomography findings; the most commonly observed finding was ground-glass appearance $(n=181 ; 27.2 \%)$. In accordance with the literature, there was primarily bilateral involvement in $153(23 \%)$ and peripheral involvement in 80 (12\%). The frequency of groundglass opacities is reported between $46-100 \%$. In another study, ground-glass opacities were detected in $88 \%$ of 919 patients and described as the most common finding (19). Ground-glass opacities are suggested to occur as a result of increased capillary blood flow, partial filling of alveoli, shifting of air due to interstitial thickening. It is described as mildly increased lung density without disruption of bronchial and vascular threshold (20). In the study group, consolidation was the second common finding in the overall CT evaluation. According to the literature, consolidation was reported in 2-64\% of COVID-19 infections. Initially, ground-glass involvement may accompany lung involvement and consolidation may supervene within 1-3 weeks which suggests progressing disease $(20,21)$. Consolidation is increased parenchymal density that occurs as a result of replacement of alveolar air with pathological fluid, cells and tissue. The bronchial and vascular structures appear indistinct (22). SARS$\mathrm{CoV}-2$ was reported to use angiotensin-converting enzyme-2 as a cell entry receptor in humans which leads to initial pulmonary damage followed by parenchymal changes (23). Lymphadenopathy was reported in 4-8 $\%$ of COVID-19 patients; although rarely seen it was associated with critical disease (21). In this study, nodular appearance/LAP was detected $50(7.5 \%)$ of the CT scan findings. Pulmonary parenchymal opacities with a diameter of $\leq 3 \mathrm{~cm}$ is defined as nodules and such nodules can be observed in viral pneumonia. Ground-glass opacity surrounding a nodule is defined as the "Halo sign" (22). Cobblestone appearance is defined as intralobular and interlobular septa accompanying ground-glass opacities. Alveolar edema and acute pulmonary damage may be due to interstitial inflammation. This appearance was reported in 5-36\% of COVID-19 patients $(22,24)$. Overall, cobblestone appearance was detected in $22(3.3 \%)$ of our findings. The reticular pattern was described as pulmonary interstitial thickenings such as inter-lobular septa and 
intra-lobular lines. The occurrence of this pattern can be associated with interstitial lymphocyte infiltration which causes interlobular septal thickening. As the disease course is prolonged, the prevalence of reticular pattern may be increased in COVID-19 patients $(21,22,25)$. In our patient group, $17(2.6 \%)$ reticular patterns were observed. Pleural thickening $(14 ; 2.1 \%)$, pleural effusion (9; $1.4 \%)$, bronchiectasis changes $(5 ; 0.8 \%)$, vascular enlargement $(4 ; 0.6 \%)$ were less frequently observed which was compatible with the literature.

\section{CONCLUSION}

Early detection, isolation and treatment are essential for control of the pandemic. Thorax CT is crucial for the early diagnosis of COVID-19. The most common CT findings include ground-glass appearance with consolidations. The findings may be variable among patients and different disease stages. In order to determine the guiding prognostic features for COVID-19, further investigation of the correlation between radiological and pathological findings is needed.

\section{ETHICAL DECLARATIONS}

Ethics Committee Approval: This study was designed and submitted to the T.R. Ministry of Health COVID-19 Scientific Research Committee and Ataturk University Medical School Clinical Research Ethics Committee for approval. Approval was granted by both committees (ethical approval no: B.30.2.ATA.0.01.00/276).

Informed Consent: All patients signed the free and informed consent form.

Referee Evaluation Process: Externally peer-reviewed.

Conflict of Interest Statement: The authors have no conflicts of interest to declare.

Financial Disclosure: The authors declared that this study has received no financial support.

Author Contributions: All of the authors declare that they have all participated in the design, execution, and analysis of the paper, and that they have approved the final version.

\section{REFERENCES}

1. Zhu N, Zhang D, Wang W, et al. A novel coronavirus from patients with pneumonia in China, 2019. N Engl J Med 2020; 382: 727-33.

2. Cui J, Li F, Shi ZL. Origin and evolution of pathogenic coronaviruses. Nat Rev Microbiol 2019; 17: 181-92.

3. Zu ZY, Jiang MD, Xu PP, Chen W, Ni QQ, Lu GM, Zhang LJ. Coronavirus Disease 2019 (COVID-19): A Perspective from China. Radiology 2020; 296: 15-25. doi:10.1148/ radiol.2020200490

4. Zhou F, Yu T, Du R, et al. Clinical course and risk factors for mortality of adult inpatients with COVID-19 in Wuhan, China: a retrospective cohort study. Lancet 2020; 28; 395: 1054-62.
doi:10.1016/S0140-6736(20)30566-3

5. Singhal, T. A Review of Coronavirus disease-2019 (COVID-19). Indian J Pediatr 2020; 87: 281-6. doi:10.1007/s12098-020-032636

6. Huang P, Liu T, Huang L, et al. Use of chest CT in combination with negative RT-PCR assay for the 2019 novel coronavirus but high clinical suspicion. Radiology 2020; 295: 22-3. doi:10.1148/ radiol.2020200330

7. Xie X, Zhong Z, Zhao W, Zheng C, Wang F, Liu J. Chest CT for typical coronavirus disease 2019 (COVID-19) Pneumonia: relationship to negative RT-PCR testing. Radiology 2020; 296: 41-5. doi:10.1148/radiol.2020200343

8. Kang Z, Li X, Zhou S. Recommendation of low-dose CT in the detection and management of COVID-2019. Eur Radiol 2020; 30: 4356-7. doi:10.1007/s00330-020-06809-6

9. Ai T, Yang Z, Hou H, et al. Correlation of chest CT and RT-PCR testing for coronavirus disease 2019 (COVID-19) in China: a report of 1014 cases. Radiology 2020; 296: 32-40. doi: 10.1148/ radiol.2020200642

10. Bai HX, Hsieh B, Xiong Z, et al. Performance of radiologists in differentiating COVID-19 from non-COVID-19 viral pneumonia at chest CT. Radiology 2020; 296: 46-54. doi:10.1148/ radiol.2020200823

11. Chung M, Bernheim A, Mei X, et al. CT imaging features of 2019 novel coronavirus (2019-nCoV). Radiology 2020; 295: 202-7. doi:10.1148/radiol.2020200230

12. $\mathrm{Xu} \mathrm{X}, \mathrm{Yu} \mathrm{C}, \mathrm{Qu}$ J, et al. Imaging and clinical features of patients with 2019 novel coronavirus SARS-CoV-2. Eur J Nucl Med Mol Imaging 2020; 47: 1275-80. doi:10.1007/s00259-020-04735-9

13. Inui $S$, Fujikawa $A$, Jitsu $M$, et al. Chest $C T$ findings in cases from the cruise ship "Diamond Princess" with coronavirus disease 2019 (COVID-19). Radiol Cardiothorac Imaging 2020; 2 : e200110. doi: 10.1148/ryct.2020200110

14. Wu Z, McGoogan JM. Characteristics of and important lessons from the coronavirus disease 2019 (COVID-19) outbreak in China: summary of a report of 72314 cases from the Chinese Center for Disease Control and Prevention. JAMA 2020; 323: 1239-42. doi:10.1001/jama.2020.2648

15. Martins-Filho PR, Tavares CSS, Santos VS. Factors associated with mortality in patients with COVID-19. A quantitative evidence synthesis of clinical and laboratory data. Eur J Intern Med 2020; 76: 97-9. doi:10.1016/j.ejim.2020.04.043

16. Petrilli CM, Jones SA, Yang J, et al. Factors associated with hospital admission and critical illness among 5279 people with coronavirus disease 2019 in New York City: prospective cohort study. BMJ 2020; 369: m1966. doi:10.1136/bmj.m1966

17. Rodriguez-Morales AJ, Cardona-Ospina JA, Gutiérrez-Ocampo E, et al. Clinical, laboratory and imaging features of COVID-19: A systematic review and meta-analysis. Travel Med Infect Dis 2020; 34: 101623. doi:10.1016/j.tmaid.2020.101623

18. Fu L, Wang B, Yuan T, et al. Clinical characteristics of coronavirus disease 2019 (COVID-19) in China: A systematic review and meta-analysis. J Infect 2020 Jun; 80: 656-65. doi:10.1016/j. jinf.2020.03.041

19. Salehi S, Abedi A, Balakrishnan S, Gholamrezanezhad A. Coronavirus disease 2019 (COVID-19): a systematic review of imaging findings in 919 patients. AJR Am J Roentgenol 2020; 215: 87-93. doi:10.2214/AJR.20.23034

20. Ye Z, Zhang Y, Wang Y, Huang Z, Song B. Chest CT manifestations of new coronavirus disease 2019 (COVID-19): a pictorial review. Eur Radiol 2020; 30: 4381-9. doi:10.1007/s00330-020-06801-0

21. Shi $H, H a n X$, Jiang N, et al. Radiological findings from 81 patients with COVID-19 pneumonia in Wuhan, China: a descriptive study. Lancet Infect Dis 2020; 20: 425-34. doi:10.1016/S1473- 
3099(20)30086-4

22. Hansell DM, Bankier AA, MacMahon H, McLoud TC, Müller NL, Remy J. Fleischner Society: glossary of terms for thoracic imaging. Radiology 2008; 246: 697-722. doi:10.1148/ radiol.2462070712

23. Xu X, Chen P, Wang J, et al. Evolution of the novel coronavirus from the ongoing Wuhan outbreak and modeling of its spike protein for risk of human transmission. Sci China Life Sci 2020; 63: 457-60. doi:10.1007/s11427-020-1637-5

24. Wu J, Wu X, Zeng W, et al. Chest CT findings in patients with coronavirus disease 2019 and its relationship with clinical features. Invest Radiol 2020; 55: 257-61. doi:10.1097/ RLI.0000000000000670

25. Ajlan AM, Ahyad RA, Jamjoom LG, Alharthy A, Madani TA. Middle East respiratory syndrome coronavirus (MERS-CoV) infection: chest CT findings. AJR Am J Roentgenol 2014; 203: 782-7. doi:10.2214/AJR.14.13021 